
\title{
Resesarch Suatere \\ Clinical Efficacy of Intramedullary Fixation vs. Plate Fixation for Femoral Intertrochanteric Fractures
}

\author{
Yu Zhou \\ Affiliated Zhongshan Hospital of Dalian University \\ Chongjun Xia \\ Affiliated Zhongshan Hospital of Dalian University \\ Xiaowei Ma \\ Affiliated Zhongshan Hospital of Dalian University \\ Xiaobing Yu ( $\square$ yuxiaobing1976@hotmail.com ) \\ Affiliated Zhongshan Hospital of Dalian University
}

\section{Research article}

Keywords: femoral intertrochanteric fractures, anatomical locking compression plate, intramedullary fixation

Posted Date: June 16th, 2020

DOI: https://doi.org/10.21203/rs.3.rs-30681/v1

License: (1) (1) This work is licensed under a Creative Commons Attribution 4.0 International License. Read Full License 


\section{Abstract \\ Background}

To compare the clinical efficacy of proximal femoral intramedullary fixation and anatomical locking compression plate in the treatment of femoral intertrochanteric fractures among the elderly.

\section{Methods}

In this retrospective study, 210 surgically treated patients with intertrochanteric fractures of the femur were analyzed and divided into groups A, B and C according to the surgical approach. Group A had interlocking compression nailing (INTERTAN) done $(n=69)$; group $B$ had $y$-III interlocking intramedullary nailing $(y-$-III) done $(n=73)$; and group $C$ had proximal femoral anatomic locking plate (PFLP) done $(n=$ 68). All patients' data were followed up completely, and their clinical outcomes were observed.

\section{Results}

Group A and B were found to perform better than group $C$ in operation time, bleeding volume, time of leaving bed, fracture healing time and hospitalization time $(P<0.05)$. Their respective incidences of postoperative complications were significantly lower than that of group $C(P<0.05)$. There were no significant differences between group $A$ and group $B(P>0.05)$. The Harris scores of groups $A$ and $B$ at 1 , 3 and 6 months after operation were significantly higher than those of group $C(P<0.05)$. There were no significant differences between group $A$ and group $B(P>0.05)$.

\section{Conclusions}

In the treatment of femoral intertrochanteric fractures, intramedullary fixation influences little on blood circulation at the fracture end, accelerates fracture healing, and enhances hip joint function, which assists patients in recovering faster postoperatively. Moreover, it enables them to gain mobility at an early stage and reduces postoperative complications of fractures. Its short-term clinical effects are better than extramedullary fixation, which should be more widely adapted clinically.

\section{Background}

The incidence of femoral intertrochanteric fractures is relatively high in the middle-aged and elderly, and the incidence in women is higher than that in men. When the disease is treated conservatively, patients require long periods of bedrest, which can lead to complications like bedsores, lower limb venous thrombosis, and hypostatic pneumonia. Thanks to the development of surgical techniques and internal fixation materials, the incidence of complications has been greatly reduced. The surgical treatment of femoral intertrochanteric fractures has become the preferred method [1]. Presently, the choice of internal 
fixation is still controversial. Common intramedullary fixation systems include compression interlocking intramedullary compression nailing system (INTERTAN) and $\mathrm{\gamma}$-III interlocking intramedullary nailing ( $\mathrm{\gamma}$-III). Common extramedullary fixation systems include proximal femoral anatomical locking plate (PFLP). Each system has its own advantages and disadvantages [2]. Therefore, we this study comparatively analyzes the clinical efficacy of INTERTAN, $Y$-III interlocking intramedullary nailing and PFLP in treating femoral intertrochanteric fractures and provides references to improve curative management.

\section{Methods}

\section{Patients}

A total of 210 patients with femoral intertrochanteric fractures who underwent surgical treatment were selected from January 2015 to December 2019. Inclusion criteria is as follows: the diagnosis of all patients was confirmed via imaging and a clear medical history of hip joint trauma. Exclusion criteria were patients with pathological fractures, blood disorders or immune system diseases. According to the operation mode, they were divided into groups. Group A was comprised of patients who underwent compression interlocking intramedullary compression nailing (INTERTAN) $(n=69)$. In group A, 33 males and 36 females aged $40-85$ years had an average age of (63.72 \pm 6.28$)$ years. Group B had patients who had $y$-III interlocking intramedullary nailing ( $(y-I I)$ done $(n=73)$. In group $B$, there were 35 males and 38 females, aged 35-84 years, with an average age of $(62.61 \pm 6.38)$ years. Group $C$ contained patients who had proximal femoral anatomical locking plate (PFLP) surgery $(n=68)$. In group $C$, there were 33 males and 35 females, aged 34-94 years, with an average age of $(64.49 \pm 6.79)$ years. There were no significant differences in the general data of the three groups of patients $(P>0.05)$, indicating that the general data of the three groups of patients were comparable.

\section{Ethical Certification}

This study was approved by the Ethics Committee of Affiliated Zhongshan Hospital of Dalian University and monitored by an independent experimental center. All patients who participated in the study gave their voluntary written informed consent.

Methods: Three groups of patients fasted with water 6 hours before operation. Antibiotics were intravenously injected 30 minutes before operation. Continuous epidural anesthesia or general anesthesia was given. Routine disinfection and paving were carried out, and fracture reduction was done under the guidance of a $\mathrm{C}$ arm.

Group A (INTERTAN): The patients were reset with traction, adduction and internal rotation in the supine position. A straight incision was made at the proximal end of the top of the ectotrochanter to cut the muscle fibers, and a rhombus awl was applied to cut the top bone to expose the medullary cavity. After the guide needle was inserted into the medullary cavity, the medullary cavity was enlarged, and the main needle was implanted under X-ray, where a Kirschner wire was positioned. When satisfactory results were 
obtained under the guidance of a $\mathrm{C}$ arm, the interlocking nail was placed in the femoral neck, and the distal femur was locked.

Group B (Y-III): The patients were reset with traction, adduction and internal rotation in the supine position. A straight incision was made at the proximal end of the top of the greater trochanter to cut the muscle fibers, and a rhombus awl was used to cut the top bone to expose the medullary cavity. After the guide needle was inserted into the medullary cavity, the medullary cavity was enlarged, the main needle was implanted under X-ray, and the Kirschner wire was positioned. When satisfactory results were obtained under the guidance of a $\mathrm{C}$ arm, the screw was pressed into the femoral neck, and the distal femoral end was locked.

Group C (PFLP): The patient was in the supine position, and the hip joint was slightly elevated. The proximal femur and greater trochanter were exposed through a lateral incision of the hip joint. After traction reset of the fracture, the steel plate and Kirschner wire were placed from the neck of the femoral head. When satisfactory results were obtained under the guidance of a C-arm, the pressure screw was fixed and tightened after installing the sleeve plate.

Antibiotics were intravenously injected 72 hours after operation to prevent infection, and low molecular weight heparin was given to prevent thrombosis for 7 days. Moreover, hip joint functional exercise was started 3 - 7 days after the operation. The operation time, bleeding volume, time to leave bed, fracture healing time, incidence of complications, and discharge time at 1, 3 and 6 months after operation were observed, and the hip joint function was evaluated via Harris scoring. All data were processed with SPSS 22.0 and tested with t-test expressed by $(x \pm s)$. $P<0.05$ was designated to be statistically significant.

\section{Results}

The operation time, intraoperative blood loss, time of leaving bed, fracture healing time and hospitalization time between group $A$ and group $B$ were significantly better than those of group $C(P<$ 0.05 , Table 1 and Fig. 1, 2, 3). The postoperative complications in group $A$ and $B$ were lower than those in group $C(P<0.05$, Table 2$)$, and the postoperative Harris score of hip joints in group $A$ and $B$ were significantly higher than that in group $C(P<0.05$, Table 3$)$.

\section{Discussion}

Femoral intertrochanteric fractures are common senile fractures that seriously affect the quality of life among elderly patients. Surgical treatment is currently the most common clinical treatment option [3] [4]. At present, the common surgical methods for treating intertrochanteric fractures of the femur include PFNA, Gamma nail, Intertan, PFLP, and dynamic hip screw (DHS). The first three embody the intramedullary system, while the latter two signify the extramedullary system. Various studies have shown that the extramedullary system is more suitable for osteoporotic unstable femoral 
intertrochanteric fractures (Evans III and above). With continuous improvements in technology, intramedullary fixation has become the mainstream modality in treatment ${ }^{[5][6]}$.

Compared with extramedullary fixation, intramedullary fixation holds the following advantages: $\otimes$ the intramedullary fixation system belongs to central fixation, which is more in line with the biomechanics of the proximal femur and can better perform femoral anatomical reduction, improving the stability of

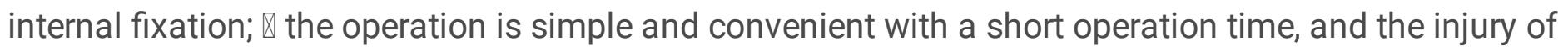
blood vessels and nerves may be greatly avoided ${ }^{[7][8][9] ; ~} \nabla$ the surgical incision is small, which protects the blood supply of the fracture end to the greatest extent and is conducive to fracture healing. The postoperative incision healing time is shorter, and the postoperative recovery is faster ${ }^{[10][11][12] ; ~} \otimes$ early mobility can greatly reduce the occurrence of postoperative complications, and hip joint functional

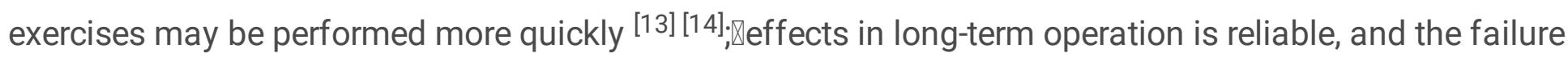
rate is relatively low with the passage of time ${ }^{[15][16][17]}$.

In this study, group A and group B outperformed group $C$ in operation time, bleeding volume, time of leaving bed, fracture healing time and hospitalization time $(P<0.05)$. The incidence of complications in group $A$ and group $B$ is significantly lower than that in group $C(P<0.05)$. The Harris score in group $A$ and group $B$ is significantly higher than that in group $C$ at 1,3 and 6 months after operation $(P<0.05)$, respectively. The results of this study prove that intramedullary fixation in treating femoral intertrochanteric fracture is evidently better than extramedullary fixation, which is consistent with previous clinical research. The main shortcomings of this study are as follows: $\mathbb{\Delta}$ subjective factors exist in the choice of surgical treatment methods for femoral intertrochanteric fractures, which may affect the evaluation of surgical efficacy; and $\otimes$ different operators have different surgical skills, which may affect surgical outcomes.

\section{Conclusion}

In regard to the treatment of femoral intertrochanteric fractures, intramedullary fixation influences little on blood circulation at the fracture end, accelerates fracture healing, and enhances hip joint function, which helps patients recover faster postoperatively. Furthermore, it allows them to benefit from early mobility and reduces the postoperative complications of fractures. Its short-term clinical effect is better than extramedullary fixation, hence, it should be adapted more widely in clinical practice.

\section{Declarations}

\section{Ethics approval and consent to participate}

This research was approved by the ethics committee of Affiliated Zhongshan Hospital of Dalian University. All participants agreed with the data and publication of the manuscript.

\section{Consent to publish}


Written informed consent was obtained from all participants.

\section{Availability of data and materials}

The data of the present study is available from the corresponding author on request

\section{Competing interests}

The authors declare that they have no competing interests.

\section{Funding}

This research did not receive any specific grant from funding agencies in the public, commercial, or notfor-profit sectors.

\section{Author contribution}

Yu Zhou: Conceptualization, Methodology,Writing- Original draft preparation. Chongjun Xia: Data curation,Software.Yu Zhou and Chongjun Xia : Visualization, Investigation. Xiaowei Ma: Supervision, Validation.Xiaobing Yu: Writing- Reviewing and Editing

\section{Acknowledgments}

We thank Dr. Tienan Wang for his professional assistance in data collection and analysis.

\section{References}

1. Bucholz RW. Rockwood and Green's Fractures in Adults: Two Volumes Plus Integrated Content Website.Rockwood, Green, and Wilkins' Fractures). Wolters Kluwer Health. 2012;22(3):309-15.

2. Chandak R, Malewar N, Jangle A, Agarwal R, Sharma M. Kekatpure ADescription of new "epsilon sign" and its significance in reduction in highly unstable variant of intertrochanteric fracture. Eur $\mathrm{J}$ Orthop Surg Traumatol. 2019;29(7):1435-9.

3. Joshi D, Dhamangaonkar AC, Ramawat S, et al. Predictors of iatrogenic lateral wall fractures while treating intertrochanteric fracture femur with the dynamic hip screw system in Indian patients. Eur $\mathrm{J}$ Orthop Surg Traumatol. 2015;25:677-82.

4. Palm H, Jacobsen S, Sonne-Holm S, Gebuhr P. Hip fracture study group Integrity of the lateral femoral wall in intertrochanteric hip fractures: an important predictor of a reoperation. $J$ Bone Joint Surg Am. 2007;89:470-5.

5. Dhamangaonkar AC, Joshi D, Goregaonkar AB, Tawari AA. Proximal femoral locking plate versus dynamic hip screw for unstable intertrochanteric femoral fractures. J Orthop Surg (Hong Kong). 2013;21:317-22.

6. Wang J, Wei J, Wang M. The risk factors of perioperative hemoglobin and hematocrit drop after intramedullary nailing treatment for intertrochanteric fracture patients.JOrthop Sci20, 163-167 
(2015).

7. O'Malley MJ, Kang KK, Azer E, et al. Wedge effect following intramedullary hip screw fixation of intertrochanteric proximal femur fracture. Arch Orthop Trauma Surg. 2015;135:1343-7.

8. Li, et al. Clinical evaluation of the Asian proximal femur intramedullary nail antirotation system (PFNA-II) for treatment of intertrochanteric fractures. J Orthop Surg Res. 2014;9:112.

9. Radcliff TA. Increased use of intramedullary nails for intertrochanteric proximal femoral fractures in veterans affairs hospitals. JBJS. 2012;94:833-40.

10. Luo P, Xu D, Guo WJ. Letter to the Editors: Comparing surgical interventions for intertrochanteric hip fracture by blood loss and operation time: a network meta-analysis. J Orthop Surg Res. 2019;14:144.

11. Spencer RF. Retraction: "Outcome of short proximal femoral nail antirotation and dynamic hip screw for fixation of unstable trochanteric fractures. A randomised prospective comparative trial" [Hip Int 2011; 21(05): 531-6]. Hip Int. 2012;22:487.

12. Liu $Y$, Sun $Y$, Fan $L$, et al. Perioperative factors associated with hidden blood loss in intertrochanteric fracture patients. Musculoskelet Surg. 2017;101:139-44.

13. Kayali $C$, Altay $T$, Kement $Z$, et al. A rare complication after intertrochanteric fracture treated with proximal femoral nail: femoral neck insufficiency fracture. Eur J Orthop Surg Traumatol. 2013;23:241-5.

14. Peyser A, Weil YA, Brocke L, Sela Y, Mosheiff R, Mattan Y, Manor O, Liebergall M. A prospective, randomised study comparing the percutaneous compression plate and the compression hip screw for the treatment of intertrochanteric fractures of the hip. J Bone Joint Surg (Br). 2007;88-B:1210-7.

15. Wu, et al. Ipsilateral femoral neck and shaft fractures fixation with proximal femoral nail antirotation II (PFNA II): technical note and cases series. J Orthop Surg Res. 2020;15:20.

16. Liu L, Sun $Y$, Wang $L$, et al. Total hip arthroplasty for intertrochanteric fracture fixation failure. Eur $J$ Med Res. 2019;24:39.

17. Sharma A, Sethi A, Sharma S. Treatment of stable intertrochanteric fractures of the femur with proximal femoral nail versus dynamic hip screw: a comparative study. Revista Bras de Ortop. 2018;53(4):477-81.

\section{Tables}

Table 1 Operation-related Information of Each Group

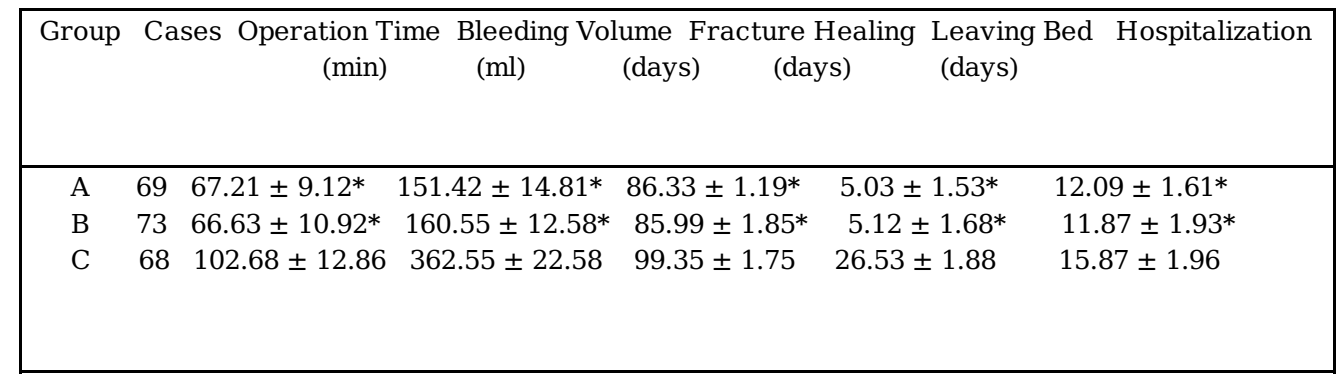


Table 2 Comparison of Surgical Complications in Each Group

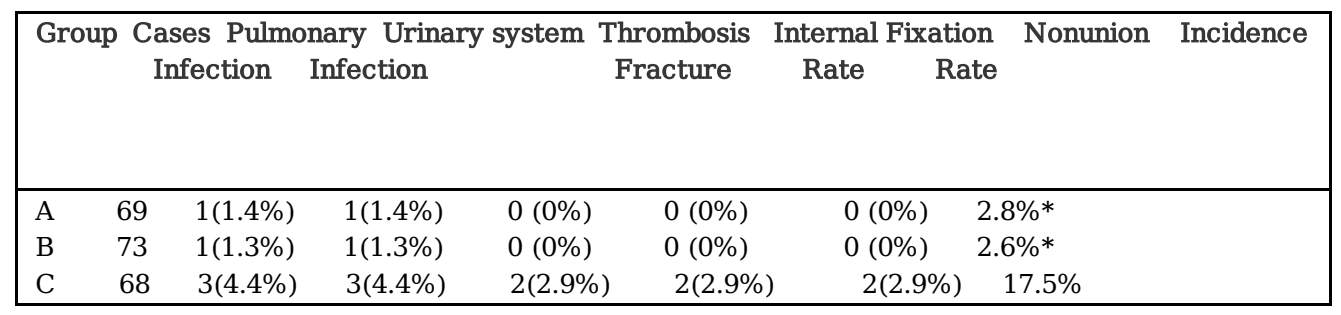

Compared with group C, group A and group B, $P<0.05$. Compared with group A and group B, $P>0.05$

Table 3 Harris Score of Hip Joint after Operation in Each Group

\begin{tabular}{|ccccc|}
\hline Group & Cases & Score 1 mth postoperation & 3 mths postoperation & 6 mths postoperation \\
\hline A & 69 & $61.18 \pm 6.84^{*}$ & $75.16 \pm 7.36^{*}$ & $84.78 \pm 8.66^{*}$ \\
B & 73 & $60.88 \pm 6.75^{*}$ & $74.86 \pm 6.96^{*}$ & $85.18 \pm 7.87^{*}$ \\
C & 68 & $51.63 \pm 5.87$ & $64.45 \pm 5.76$ & $73.69 \pm 7.76$ \\
\hline
\end{tabular}

Compared with group C, group A and group B, $P<0.05$. Compared with group A and group B, $P>0.05$

\section{Figures}
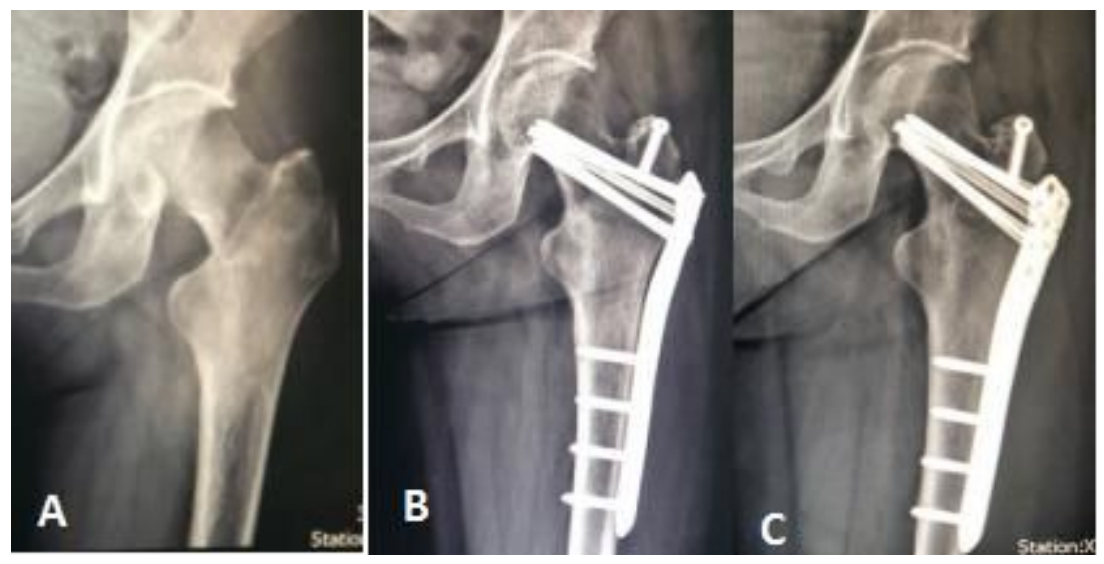

Figure 1 


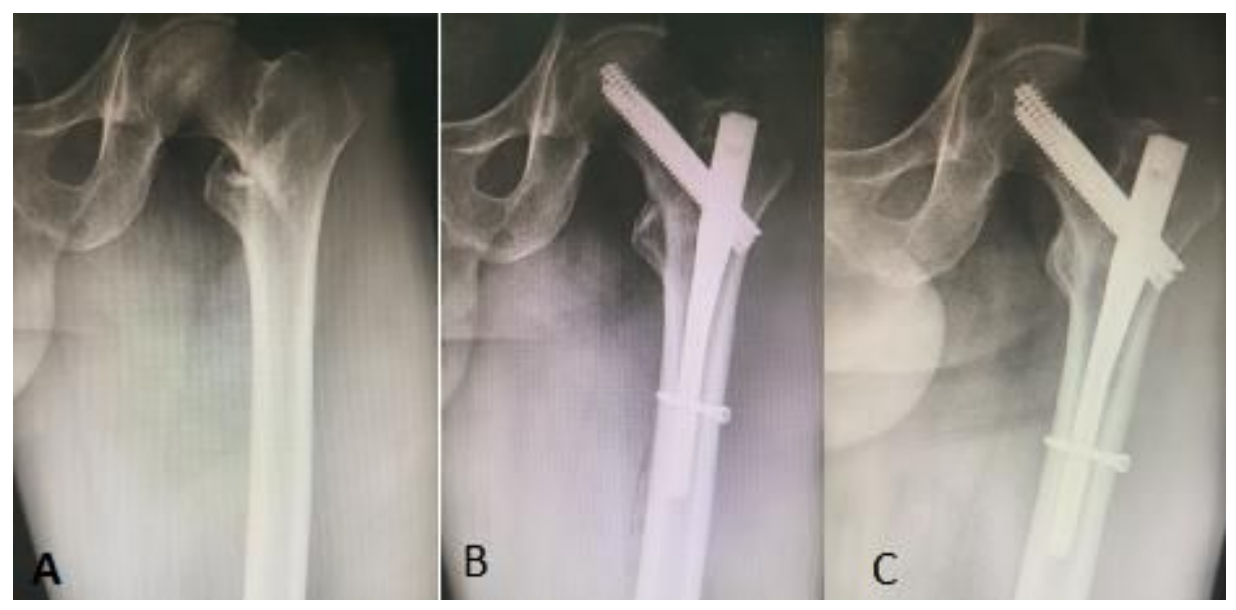

Figure 2

A 79-year-old man with left intertrochanteric femoral fracture fixed with INTERTAN internally . A, preoperative X-ray image. B, postoperative X-ray image.C,one year postoperative X-ray image

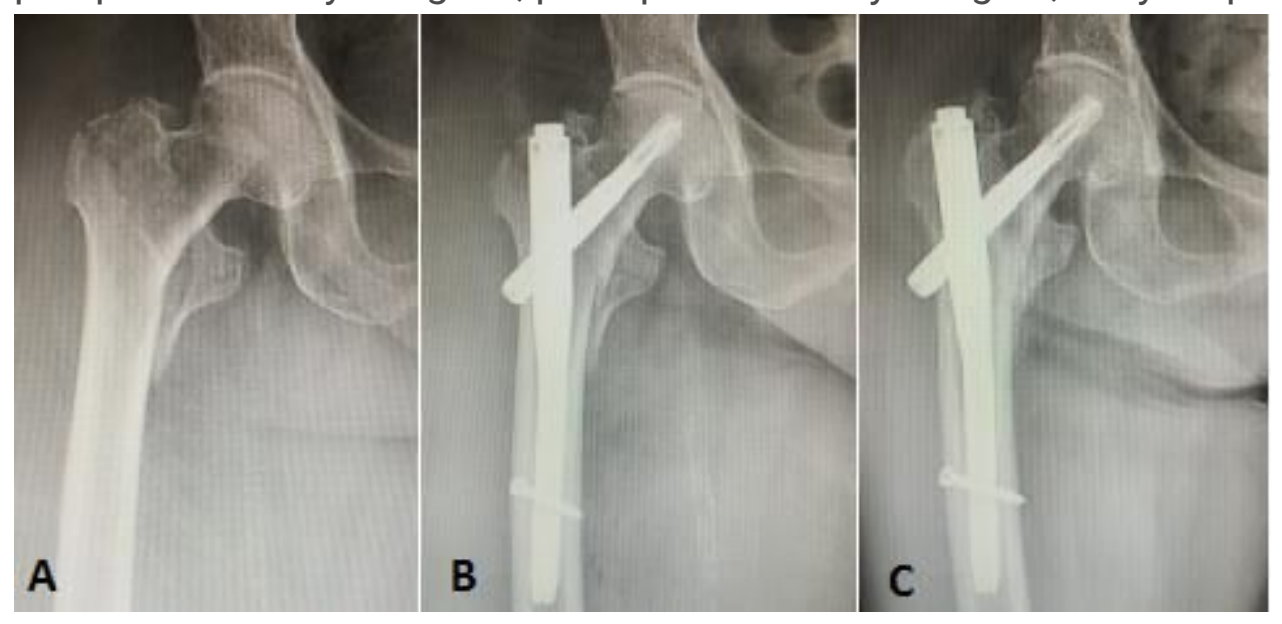

\section{Figure 3}

A 84-year-old woman with right intertrochanteric femoral fracture fixed withy-III internally . A, preoperative X-ray image. B, postoperative $\mathrm{X}$-ray image.C,one year postoperative $\mathrm{X}$-ray image 